\title{
Primary reconstruction of pharyngo-oesophageal defects in oncologic patients
}

\author{
Palencar $^{1}$, Kroslak $\mathbf{M}^{2}$, Fedeles $\mathbf{J}^{1}$ \\ Department of Plastic Surgery, Medical School, Comenius University, Bratislava, Slovakia. \\ d.palencar@mail.t-com.sk
}

\begin{abstract}
Objectives: Majority of patients that undergo total or partial removal of the hypopharynx and cervical oesophagus are oncologic patients. Optimal management of head and neck malignancies requires multimodal therapy including surgical ablation, reconstruction, and adjuvant oncologic therapy. Despite aggressive surgical and adjuvant therapy, a five-year survival rate is achieved only in $25-35 \%$.

Methods: In the presented retrospective study, the choice of reconstructive method was influenced by type, length and extent of the defect, and condition of patient. The flap was indicated when the defect not allowed for primary suture of the hypopharynx and/or cervical oesophagus. Two-team approach was used.

Results: The study was based on the data of ten patients. Radial forearm flap was used in 8 cases; pectoralis major myocutaneous flap was used in 3 patients, and ALT perforator free flap in 1 case. A total of 12 flaps were used for 10 patients. Two patients developed free flap necrosis. These necrotic flaps were substituted with pedicled pectoralis major myocutaneous flaps.

Conclusions: The primary reconstruction of the pharyngo-oesophageal defects could be the method of choice. For the partial defects, the best choice could be a radial forearm free flap. For circumferential defects, jejunal flap could be the best. The pectoralis major pedicled flap could be a safe procedure for elderly patients with multiple medical problems (Tab. 6, Fig. 2, Ref. 34). Full Text in PDF www.elis.sk.

Key words: pharynx reconstruction, pharyngo-oesophageal reconstruction, ALT perforator flap, free radial forearm flap, pectoralis major flap.
\end{abstract}

Surgical resection of the hypopharynx and cervical oesophagus may lead to severe functional deficits, and it may prevent the patient's peroral intake of solid food and liquids. This usually applies to patients with locally advanced laryngeal or hypopharyngeal malignancy. The treatment of these patients involves resection and reconstruction followed by radiotherapy. Surgical resection and reconstruction of the hypopharynx in patients suffering from advanced oncological disease is a challenge for surgeons, anaesthesiologists, nurses, and represents serious stress for patient.

Resection of a small part of the hypopharynx or cervical oesophagus allows for primary suture of the defect, and it results in only minimal and temporary changes. Extensive resections require difficult reconstructions, often associated with free transfer of tissues. Despite the extensive surgical therapy and aggressive adjuvant therapy, five-year survival of patients in stage III or IV of head and neck malignancies is only $25-35 \%(1-3)$.

Nowadays, the most favoured method is that of one-stage reconstruction of gastrointestinal tract continuity. The reconstructions of hypopharynx and cervical oesophagus have progressed

${ }^{1}$ Department of Plastic Surgery, Medical School, Comenius University, Bratislava, Slovakia, and ${ }^{2}$ Department of Otorhinolaryngology, Medical School, Comenius University, Bratislava, Slovakia

Address for correspondence: D. Palencar, MD, PhD, Dept Plastic Surgery, UniHospital Bratislava, Ruzinovska 6, SK-826 06 Bratislava, Slovakia. Phone: +421.2 .48234886$ from multiple-stage procedures using pedicle skin flaps (4-8) to the use of pedicle fasciocutaneous, myocutaneous and visceral flaps (9-15). The advent of microsurgical free flaps has allowed for transferring the best available tissue for reconstructions. The microsurgical technique has also improved the reliability and reduced the complication rate (16-25).

The aim of the study was to evaluate retrospectively the outcome after pharyngo-oesophageal cancer in terms of complications of surgery, success of reconstruction methods, and survival rate.

\section{Methods}

The primary reconstruction of pharyngo-oesophagus in oncologic patients was done in cooperation with ENT surgeons. Wide defects not allowing for primary suture were an indication for reconstruction with the use of flap. All the patients were in stage III or IV of head and neck cancer. The indication of the use of flap was fully in oncosurgeon's competence. His decision was based on preoperative examinations (local state, state of lymphatic nodes, ultrasound, X-ray, CT, pharyngoscopy, biopsy results) and preoperative findings. The type of flap was chosen by plastic surgeon. The operation was done under a two-team approach. Oncologic team started the operation (tumour resection, neck dissection and recipient vessels preparation was done - should the free flap be planned). Meanwhile, the reconstructive team started raising the flap in order to minimize the operative time. 
A pedicle or free flaps were used for reconstruction of pharyngo-oesophageal defects. Pectoralis major myocutaneous flap was used as pedicled flap, while free flaps included radial forearm flap and ALT (anterolateral thigh) perforator flap.

The indications for using flap reconstruction included defects of posterior and/or lateral walls of pharynx, defects of more than $1 / 2$ of pharynx circumference, defects of soft palate extending down to the posterior and/or lateral wall of hypopharynx, circular defects of pharynx, and all named defects that extended down to the cervical part of the oefophagus. The criteria decisive for the type of reconstruction included recipient vessels condition, extent of defect, preoperative donor site condition, age and general condition of a patient, illnesses of the patient that could possibly react with anaesthesiological management and operation time.

Superior thyroid artery and internal jugular vein were used as recipient vessels in a majority of free flap cases. When these vessels were not suitable counterlateral vessels were explored or transversal colli artery and superficial veins of the neck were used. When the tumour or metastatic process afflicted the recipient vessels, and when no vessel was suitable for anastomosis, a decision for pedicle flap was made.

Age and general state of patients affected the selection of reconstructive method, namely in relation to the suitable duration of surgery. When the classification of a patient was ASA 3 or more the shortest possible reconstructive method was indicated (pedicled pectoralis major flap).

\section{Patients}

The retrospective study analysed ten patients, who had undergone operations in 1998-2004. All of them were male patients with mean age of 57 years (44-62). All patients were in stage III or IV of head and neck carcinoma. All types of flaps and numbers are shown in Table 1. All patients had epidermoid carcinoma of pharynx.

When no complications occurred, the patients started the peroral intake of food on postoperative day 14, and adjuvant therapy started 4 weeks after the operation.

Radial forearm flap was used most frequently ( 8 patients). Both pectoralis major myocutaneous flap and ATL perforator flap were used for primary reconstruction. After the radial forearm flap failure, pectoralis major flap was used as a secondary flap in two cases.

\section{Results}

The study evaluated flap vitality, formation of fistulas, period of time from operation up to restoration of peroral intake, starting time of adjuvant radiotherapy, development of dysphagia and strictures, and survival of patients.

Twelve flaps were used for 10 patients. Three of them were pedicled flaps, namely musculus pectoralis myocutaneous flap. Out of free flaps, radial forearm flaps were used 8 times while ALT perforator flap was used once. In two cases of free flap failure, necrotic flap was replaced by a pedicle myocutaneous flap from musculus pectoralis major. Subsequent recovery was free of complications. Remaining flaps, i.e. either pedicle flaps or free flaps
Tab. 1. Patients.

\begin{tabular}{cccccc}
\hline Patient & Age & Preop RAT & Flap & Flap vitality & Sec flap \\
\hline 1 & 60 & no & radial forearm & good & - \\
2 & 62 & no & radial forearm & total loss & pectoralis major \\
3 & 62 & no & radial forearm & good & - \\
4 & 55 & no & radial forearm & total loss & pectoralis major \\
5 & 51 & no & pector.major & good & - \\
6 & 61 & no & radial forearm & good & - \\
7 & 59 & no & radial forearm & good & - \\
8 & 44 & no & radial forearm & good & - \\
9 & 59 & no & radial forearm & good & - \\
10 & 59 & no & ALT & good & - \\
\hline
\end{tabular}

Tab. 2. Flap vitality.

\begin{tabular}{lccc}
\hline Flap & Amount & Necrosis & $\%$ of Success \\
\hline Pectoralis major $\mathrm{m}$. & 3 & - & $100 \%$ \\
Radial forearm flap & 8 & 2 (severe) & $75 \%$ \\
ALT & 1 & - & $100 \%$ \\
Free flaps all together & 9 & 2 & $78 \%$ \\
\hline
\end{tabular}

Tab. 3. The beginning of per os intake.

\begin{tabular}{lcc}
\hline Group of patients & Amount & $\begin{array}{c}\text { Per os from the primary } \\
\text { operation }\end{array}$ \\
\hline Patients without early complications & 8 & 2 weeks \\
Patients with flap necrosis & 2 & 4 weeks \\
All patients together & 10 & 17,4 days \\
\hline
\end{tabular}

healed without any difficulties or necroses. In respect of vitality or flap necrosis, the success rate for pedicle flaps was $100 \%$, while for free flaps, the rate was $78 \%$ (Tab. 2) (Figs 1 and 2).

Should no complication occur, on day 14 after surgery, the patients started to take postsurgical nutrition per os. An average period of time from reconstruction to the beginning of peroral intake was 17.4 days (Tab. 3).

As part of standard oncological routine, the patients with pharyngeal carcinoma start their radiotherapy 3-4 weeks after the primary resection of tumour. According to the usage of flap in reconstruction of pharyngeal defects, in case of uncomplicated procedure, we indicated radiotherapy to be started 4 weeks after the resection. A delay to the introduction of adjuvant therapy was caused by flap necrosis. In two cases with flap necrosis, a reoperation on postoperative day 14 with subsequent four-week surgical treatment was necessary. These two patients could begin their radiotherapy 6 weeks after the primary operation.

Depending on whether the monitored complications occurred up to day 14 days after the reconstruction of pharyngo-oesophagus,

Tab. 4. Late complications.

\begin{tabular}{lccc}
\hline Patients & Stricture & Local recurrence & Distant metastases \\
\hline 10 & $1(10 \%)$ & $3(30 \%)$ & $4(40 \%)$ \\
\hline
\end{tabular}

Tab. 5. Survival of patients.

\begin{tabular}{lc}
\hline Total number of patients & 10 \\
\hline Without recurrence for more than 5 years & $3(30 \%)$ \\
Exitus in the first year (1x recurrence, 1x distant mts) & $2(20 \%)$ \\
Exitus in the second year (1x recurrence, 2x distant mts) & $3(30 \%)$ \\
Exitus in the third year (1x recurrence, 1x distant mts) & $2(20 \%)$ \\
\hline
\end{tabular}




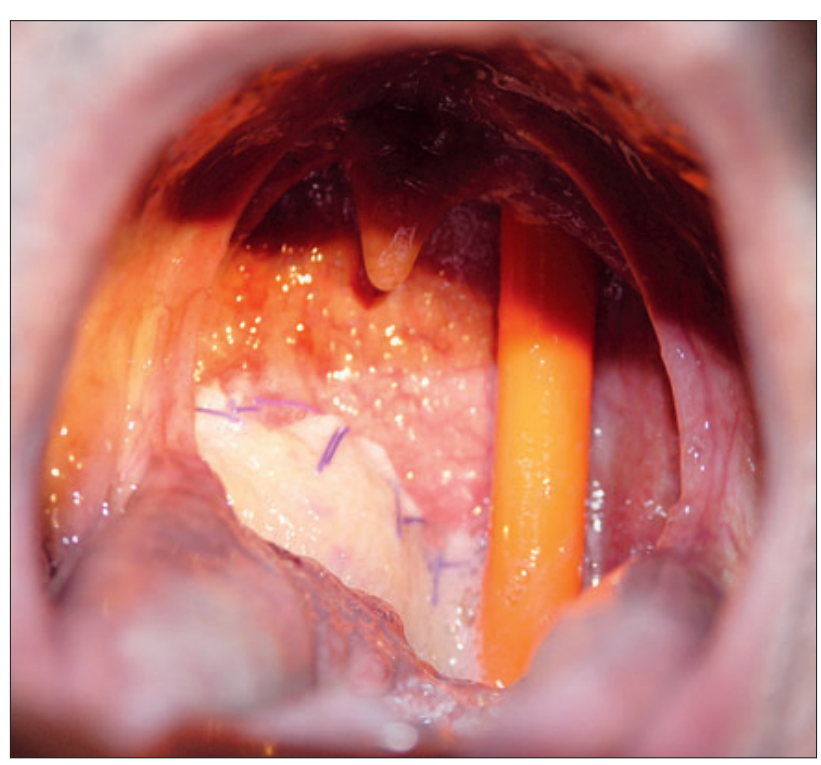

Fig. 1. View into the buccal cavity during on day 7 after surgery. On the back of oropharynx, a radial forearm flap can be seen.

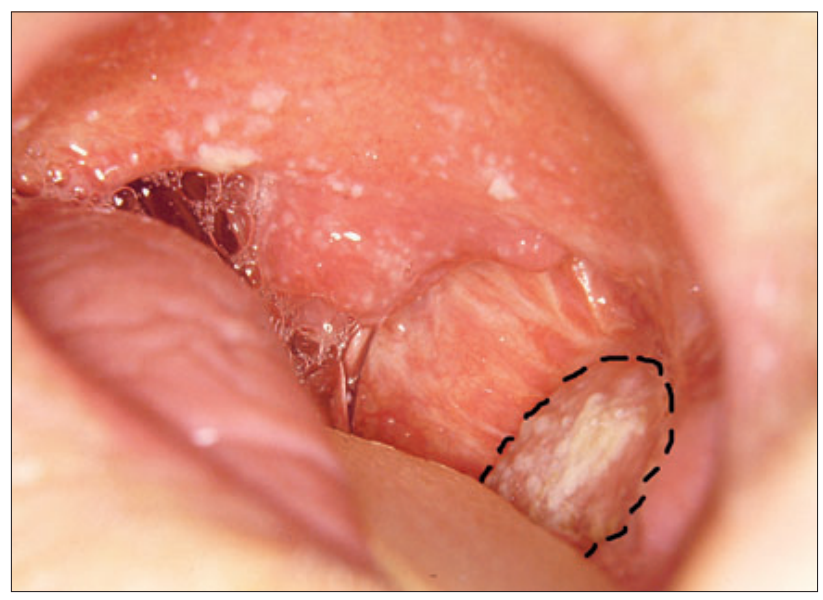

Fig. 2. View into the buccal cavity 3 months after the operation. In the area after resection of the left tonsil, the radial forearm flap extending to hypopharynx is labelled.

i.e prior to adjuvant treatment or after it, they were classified as being early or late. Flap necrosis belongs among early complications. Necrosis of two radial flaps developed into fistulas with purulently mucinous secretion (Tab. 2).

Late complications (Tab. 4) included relapse of the primary disease and strictures of the reconstructed section associated with dysphagia and fistulas. A stricture occurred in one patient. It was caused by oedema of excessive flap resulting in nourishment leaking into airways. The situation required endoscopic LASER resection of the excessive flap. Right strictures with a contraction in lieu of flap-hypopharynx anastomosis did not occur. Two months after the operation, one patient with a primary reconstruction developed a saliva fistula which healed spontaneously in four weeks. The disease did not recur and the cause of the fistula has stayed unclear.
Relapse of the primary disease was evaluated in term of local recurrence and distant metastasis. Out of 10 patients, two had local recurrence (one of whom died later due to generalization of the process). Three of ten patients died due to development of distant metastases (brain and lungs) while no local recurrence was recorded.

Survival of patients: The patients were monitored from up to 10 months to 7.5 years (average 3 years). Three patients of ten lived for more than 5 years without any signs of local recurrence or distant metastases. Two patients died up to 12 months from the primary operation due to local recurrence or distant metastases. Three patients died during the second year after primary operation due to distant metastases. The other two patients died in the third year due to distant metastases and local recurrence. Out of 10 patients, an overall of $50 \%$ of patients died due to recurrence or distant metastases up to 2 years. Out of 10 patients, $30 \%$ lived for more than 5 years after primary resection and reconstruction (Tab. 5).

\section{Discussion}

The safety of reconstructive method is one of the most important factors. Low amount of complications, low necessity of reoperations and no delay to introducing the subsequent oncologic therapy are further conditions for a good reconstructive algorithm in oncologic patient. The vitality of flap and presence of partial or complete flap necrosis play the major role in the safety of hypopharyngeal reconstruction. In pedicled flaps, with the use of ideal operative technique and preoperative doppler detection of flap pedicle, a high success rate of flap vitality can be achieved. In the study, musculus pectoralis major pedicled myocutaneous flap was used with a success rate of $100 \%$. It was reserved for patients who could not undergo time-consuming free tissue transfer, or used as a second flap in case of failure of the free flap. Musculus pectoralis major flap showed to be a safe flap for covering partial or even complete hypopharyngeal defects. Its another advantage lies also in the fact that when compared to skin island, a muscle can be removed to a greater extent, whereby the excess of muscle can possibly cover the defect of soft tissues in the neck, and thus the necessity of the second flap disappears (26).

In the study, nine free flaps were used. After the thrombosis of venous anastomoses, two of them failed, and it was necessary to replace them on postoperative day 14 with a pedicled flap from musculus pectoralis major. The success rate of free transfers was $78 \%$. Literature presents the percentage of necrosis of free flaps, used in reconstruction of hypopharynx and neck oesophagus, namely as $2.4-12 \%(27-31)$.

Patients with advanced pharyngeal malignancy are often cachectic and anaemic especially due to their inability of peroral intake of solid nourishment, even before the operation. For that reason, one of the most important factors of reconstruction success rate of a hypopharynx is the time from operation up to the introduction of peroral food intake. In the study, an average time up to introducing solid nourishment intake was 17.4 days from primary operation. Majority of authors report the latter interval to be 2 to 3 weeks $(27,29-32)$. 
Of early complications, only flap necrosis occurred. Infection in site of resection and reconstruction was not monitored.

The beginning of adjuvant radiotherapy is usually scheduled for patients with malignancy of pharyngo-oesophagus in the third or fourth week after the resection of tumour. In the study, the radiotherapy was planned to take place four weeks after the operation. The four-week interval after the reconstruction was set according to clinical experience with delay to pedicle flaps, and according to the experimental study of Cordeiro et al (33) who proved that the ligation of free flap pedicle in the neck area did not have any effect on vitality of flap four weeks after the flap transfer. In $80 \%$ of patients the time plan was kept, in $20 \%$ of cases radiotherapy was postponed to the sixth week after the primary operation due to flap necrosis and due to the coverage of substitutional flap defect from musculus pectoralis major. The delay of radiotherapy in two patients out of ten is related to the safety of reconstructive method.

Of late complications, strictures of distal anastomoses, and leaking of nourishment into the larynx (if the larynx was maintained) was most commonly reviewed in literature. In this study, a stricture occurred in one patient out of ten (10\%). However, this stricture was "fake". It was connected to dysphagia after radiotherapy. The stricture was caused by massive oedema of radial forearm flap, which obstructed the hypopharynx, where the solid nourishment leaked into the patient's airways. This problem was resolved by LASER resection of the excessive flap. The occurrence of strictures in literature is mainly described in circular defects of hypopharynx, and it is more frequent in association with the use of skin flaps in a comparison to free jejunum. Cho et al. recommends two V-flaps on the distal side of a flap, which fall into the cuts in the mucous tissue of hypopharynx and oesophagus (29). Therefore, the circumference of anastomosis expands, and the line of a circular cicatrix stops. Harii recommends making a wave line in the flapmucous contact (34). Nakatsuka indicates strictures in the use of jejunum in $9 \%$, and in the use of radial forearm flap in $39 \%$ (30).

In case histories, a five year survival of patients with advanced hypopharyngeal malignancy is listed in only $25-35 \%$ of cases (13 ). In the submitted study, the longest interval of a patient's monitoring was 7.5 years. In $30 \%$ of cases, the survival exceeds five years, in $20 \%$ of studied patients, the survival was up to three years, in other $30 \%$ it is up to two years, and in $20 \%$ it is up to one year.

\section{Conclusion}

The treatment of patients with head and neck malignancy is a challenge for all involved medics. The location of hypopharynx is so critical that after the resection of an extensive tumour, there emerges a problem of functional reconstruction which, additionally, after a certain period, has to resist the aggressive adjuvant therapy.

The primary reconstruction is a method of choice. Nowadays, one-stage reconstructions which do not delay oncologic therapy are favoured. Primary reconstruction allows early introduction of peroral food intake, it neither delays subsequent oncologic therapy, nor burdens a patient with several operations and necessity of diet for a long period of time. In primary operation, the proportions of the reconstructed area are well-arranged, not changed by the
Tab. 6. Indications of particular flaps in the reconstruction of pharyngo-oesophagus.

\begin{tabular}{lcccc}
\hline & $\begin{array}{c}\text { Musculus } \\
\text { pectoralis } \\
\text { major }\end{array}$ & $\begin{array}{c}\text { Radial } \\
\text { forearm } \\
\text { flap }\end{array}$ & Jejunum & ALT \\
\hline Oropharynx and nasopharynx & + & +++ & & \\
Circular hypopharynx & + & + & +++ & + \\
Partial hypopharynx & + & +++ & + & + \\
Old and weak patients & +++ & + & & + \\
\hline$+++:$ method of choice, & & & & \\
$+:$ can be used under certain conditions & & & &
\end{tabular}

previous operations or radiotherapy. This has strong influence on lower ing the presence of complications.

When selecting the reconstruction method, the patient's general state, extent of defect (its circumference and length), presence of fistulas and dysphagia difficulties after previous reconstruction procedures should be taken into consideration. The selection of flap should be based on criteria as follows (Tab. 6):

1. To reconstruct circumferential defects of hypopharynx by the free transfer of jejunum. Mucous membrane-mucous membrane suture is more resistant to the formation of fistulas in comparison to skin flaps.

2. Partial defects of pharynx: In the study, this defect was reconstructed with free radial forearm flap. This one has several advantages including constant anatomy, easy removal, double-team approach, lower morbidity of the donor site (compared to jejunal flap), long pedicle with sufficient calibre of vessels.

3 . Long and relatively narrow defects of hypopharynx or defects stretching from oropharynx to neck oesophagus: A proper solution for these defects could be a skin flap, which is easily modelled and thin. Here a radial forearm flap comes into consideration. A perforated flap from thigh - ALT (anterolateral thigh flap) could also be applied. The advantage of ALT flap is that it does not sacrifice any other important artery and it has got minimal, almost no morbidity of donor site. For thin patients, ALT flap could be used as an alternative to radial forearm flap, mostly for the possibility of removing it even with fascia which can be used as a second layer of hypopharyngeal anastomosis, by which the incidence rate of fistulas could be lowered. The main disadvantage of ALT flap is the preparation on the level of perforators. Therefore, the work is intended for an experienced and well-trained microsurgeon.

4. Another criterion for selecting the reconstructive method is the patient's general state of health. Free tissue transfer is timeconsuming; therefore, it is reserved for patients in good condition. Patients who have extremely advanced malignancy are weakened by the primary illness, plus they have other illnesses concomitant to the primary one (atherosclerosis, diabetes mellitus). They should be reconstructed by a less time-consuming method, as well as by a method that is more secure in term of success rate and flap vitality. This category includes pedicle myocutaneous flaps from torso, mostly pectoralis major flap.

An application of a two-team approach shortens the time of operation. At the same time it burdens neither a patient nor a surgeon and assistants with such a demanding and long performance. This approach positively affects the quality of work while the spe- 
cialisation of experts is taken into consideration. After the ablative part of the operation, done by an onco-surgical team is finished, the reconstructive team experienced with microsurgery technique takes over. Free transfer of tissues is today a method of choice; pedicled flaps should be reserved for high-risk patients and secondary cases.

An indication for the oncologic part of the operation is fully in oncosurgeon's competence, while the plastic surgeon indicates the reconstructive procedure in case of extensive resection. An extensive resection with subsequent reconstruction of a defect by a flap can improve the functional outcomes of the operation especially in relation to the intake of full nourishment including solid food.

\section{References}

1. Guillamondegui OM, Meoz R, Jesse RH. Surgical treatment of squamous cell carcinoma of the pharyngeal walls. Am J Surg 1978; 136 (4): 474-746

2. Stell PM, Missotten F, Singh SD, Ramadan MF, Morton RP. Mortality after surgery for hypopharyngeal cancer. Br J Surg 1983; 70 (12): 713-718.

3. Surkin MI, Lawson W, Biller HF. Analysis of the methods of pharyngoesophageal reconstruction. Head Neck Surg 1984; 6 (5): 953-970.

4. Bakamjian VY. A two-stage method for pharyngoesophageal reconstruction with a primary pectoral skin flap. Plast Reconstr Surg 1965; 36: 173-184.

5. Bakamjian VY, Long MD, Rigg RB. Experience with the medially based deltopectoral flap in reconstructive surgery of the head and neck. Br J Plast Surg 1971; 24 (2): 174-183.

6. Conley JJ. Management of pharyngostome, esophagostome and assotiated fistulae. Ann Otol Rhinol Laryngol 1956; 65 (1): 76-91.

7. Conley J. One-stage reconstruction of pharynx and cervical esophagus with chest flap. Bull N Y Acad Med 1969; 45 (4): 951-957.

8. Wookey H. The surgical treatment of carcinoma of the hypopharynx and the oesophagus. Br J Surg 1948; 35 (139): 249-266.

9. Ariyan S. Further experience with the pectoralis major myocutaneous flap for immediate repair of defects from excision of head and neck cancers. Plast Reconstr Surg 1979; 64 (5): 605-612.

10. Baek SM, Lawson W, Biller HF. Reconstruction of hypopharynx and cervical oesophagus with pectoralis major island myocutaneous flap. Ann Plast Surg 1981; 7 (1): 18-24.

11. Demergasso F, Piazza MV. Trapezius myocutaneous flap in reconstructive surgery for head and neck cancer: An original technique. Am J Surg 1979; 138 (4): 533-536.

12. Lees W. Colonic replacement after pharyngolaryngectomy. Br J Surg 1967; 54 (6): 541-547.

13. Silver CE. Reconstruction after pharyngolaryngectomy-esophagectomy. Am J Surg 1976; 132 (4): 428-434.

14. Spiro RH, Bains MS, Shah JP, Strong EW. Gastric transposition for head and neck cancer: A critical update. Am J Surg 1991; 162 (4): 348-352.

15. Theogaraj SD, Merrit WH, Acharya G, Cohen IK. The pectoralis major myocutaneous island flap in single-stage reconstruction of the pharyngoesophageal region. Plast Reconstr Surg 1980; 65 (3): 267-276.

16. Anthony JP, Singer MI, Mathes SJ. Pharyngoesophageal reconstruction using the tubed free radial forearm flap. Clin Plast Surg 1994; 21 (1): $137-147$.
17. Coleman JJ III, Tan KC, Searles JM, Hester TR, Nahai F. Jejunal free autograft: Analysis of complications and their resolution. Plast Reconstr Surg 1989; 84 (4): 589-595.

18. Endo T, Nakayama Y. Pharyngoesophageal reconstruction: A clinical comparison between free tensor fasciae latae and radial forearm flaps. J Reconstr Microsurg 1997; 13 (2): 93-97.

19. Fisher SR, Cameron R, Hoyt DJ, Cole TB, Seigler HF, Meyers WC. Free jejunal interposition graft for reconstruction of the esophagus. Head Neck 1990; 12 (2): 126-130.

20. Jurkiewicz MJ. Vascularised intestinal graft for reconstruction of the cervical esophagus and pharynx. Plast Reconstr Surg 1965; 36 (5): 509-517.

21. Reece GP, Bengtson BP, Shusterman MA. Reconstruction of the pharynx and cervical esophagus using free jejunal transfer. Clin Plast Surg 1994; 21 (1): 125-136.

22. Reece GP, Shusterman MA, Miller MJ, Kroll SS, Robb GL, Baldwin BJ, Luethcke DR. Morbidity and functional outcome of free jejunal transfer reconstruction for circumferential defects of the pharynx and cervical esophagus. Plast Reconstr Surg 1995; 96 (6): 1307-1316.

23. Seidenberg B, Rosenak S, Hurwitt ES, Som ML. Immediate reconstruction of the cervical esophagus by a revascularised isolated jejunal segment. Ann Surg 1959; 149 (2): 162-171.

24. Shangold LM, Urken ML, Lawson W. Jejunal transplantation for pharyngoesophageal reconstruction. Otolaryngol Clin North Am 1991; 24 (6): 1321-1342.

25. Silver CE, Cusamano RJ, Fell SC, Strauch B. Replacement of upper esophagus: Results with myocutaneous flap and with gastric transposition. Laryngoscope 1989; 99 (8 Pt1): 819-821.

26. Disa JJ, Cordeiro PG. Reconstruction of the hypopharynx and cervical esophagus. Clin Plast Surg 2001; 28 (2): 349-360.

27. Lydiatt WM, Kraus DH, Cordeiro PG, Hidalgo DA, Shah JP. Posterior pharyngeal carcinoma resection with larynx preservation and radial forearm free flap reconstruction: a preliminary report. HeadNeck 1996; 18(6): 501-505.

28. Hidalgo DA, Disa JJ, Cordeiro PG, Hu QY. A review of 716 consecutive free flaps for oncologic surgical defects: refinement in donor-site selection and technique. Plast Reconstr Surg 1998; 102 (3): 722-734.

29. Cho BC, Kim M, Lee JH, Byun JS, Park JS, Baik BS. Pharyngoesophageal reconstruction with a tubed free radial forearm flap. J Reconstr Microsurgery 1998; 14 (8): 535-540.

30. Nakatsuka T, Harii K, Asato H, Ebihara S, Yoshizumi T, Saikawa M. Comparative evaluation in pharyngo-oesophageal reconstruction: radial forearm flap compared with free jejunal flap. A 10-year experience. Scand J Plast Reconstr Surg Hand Surg 1998; 32 (3): 307-310.

31. Chen HC, Tang YB. Microsurgical reconstruction of the Esophagus. Semin Surg Oncol 2000; 19 (3): 235-245.

32. Endo T, Nakayama Y. Pharyngoesophageal reconstruction with a tensor fasciae latae free flap. Plast Reconstr Surg 1995; 95 (2): 400-405.

33. Cordeiro PG, Santamaria E, Hu QY, DiResta GR, Reuter VE. The timing and nature of neovascularization of jejunal free flaps: an experimental study in a large animal model. Plast Reconstr Surg 1999; 103 (7): 1893-901.

34. Harii K, Ebihara S, Ono I, Saito H, Terui S, Takato T. Pharyngoesophageal reconstruction using a fabricated forearm free flap. Plast Reconstr Surg 1985; 75 (4): 463-476.

Received April 11, 2011. Accepted January 20, 2013. 